\title{
Polymyositis and Hepatitis Concurrent with Breast Cancer
}

\author{
Deniz Yamac ${ }^{a}$ Nazan Gunel ${ }^{a}$ Berna Goker ${ }^{b}$ Ugur Coskun ${ }^{a}$ Ozlem Erdem ${ }^{c}$ \\ Gulen Akyolc Seren Ozenirler ${ }^{d}$
}

Departments of a Medical Oncology, ${ }^{b}$ Rheumatology, ${ }^{c}$ Pathology and ${ }^{d}$ Gastroenterology, Gazi University Medical School, Ankara, Turkey

\section{Key Words}

Breast cancer · Polymyositis · Hepatitis · Paraneoplastic syndrome

\begin{abstract}
Objective: To present a rare case of breast cancer associated with both inflammatory muscle disease and liver disease as a paraneoplastic syndrome. Clinical Presentation and Intervention: A woman with breast cancer presented with elevated liver enzymes and progressive proximal muscle weakness. Liver biopsy was consistent with hepatitis and muscle biopsy revealed myositis. The start of corticosteroid therapy was followed by relief of the myopathic symptoms and regression of hepatitis histopathologically. Conclusion: A case of polymyositis and hepatitis associated with breast cancer and their flare-up with recurrence of malignancy is presented. In this case, the temporal relation with malignancy following its concurrent remission and relapse suggests a paraneoplastic mechanism.
\end{abstract}

Copyright $\odot 2004$ S. Karger AG, Basel

\section{Introduction}

It is well accepted that patients with dermatomyositis and polymyositis have an increased risk of developing malignancy [1]. The overall odds ratio for developing a malignancy has been reported to be 4.4 for patients with dermatomyositis and 2.1 for those with polymyositis with risk before and after onset for the disease [2]. The activity of myositis may appear linked to that of the malignancy supporting the validity of an association [1]. The most common cancers occurring among female patients with idiopathic inflammatory myopathies are breast and gynecological cancers $[3,4]$. A variety of autoimmune conditions including autoimmune hepatitis have been reported in association with polymyositis. In this report, we present a case of polymyositis and hepatitis associated with breast cancer.

\section{Case Report}

A 57-year-old woman with bilateral breast cancer (infiltrating ductal carcinoma, right T2N1M0 and left T1N0M0) was admitted to the Oncology Service for adjuvant treatment 4 weeks after bilateral mastectomy. The tumor was grade 2 in the right and left breasts. ER was strongly positive. Her only complaint was fatigue which began prior to the surgery. Her past medical history included an accident a year previously which resulted in a crush injury to the pelvis, and

Dr. Deniz Yamac

Mertler sokak 31/5, Şenyuva

TR-06510 Ankara (Turkey)

Tel. +90 $3122141000 /$ ext. 5828, Fax +90 3122124647

E-Mail dyamac@gazi.edu.tr
Fax + 41613061234 E-Mail karger@karger.ch www. karger.com
1011-7571/04/0133-0171\$21.00/0

Accessible online at: www. karger.com/mpp 
Fig. 1. Macrovesicular steatosis, sinusoidal inflammation, mild focal necrosis in the liver (a) and perivascular inflammation in the muscle (b).
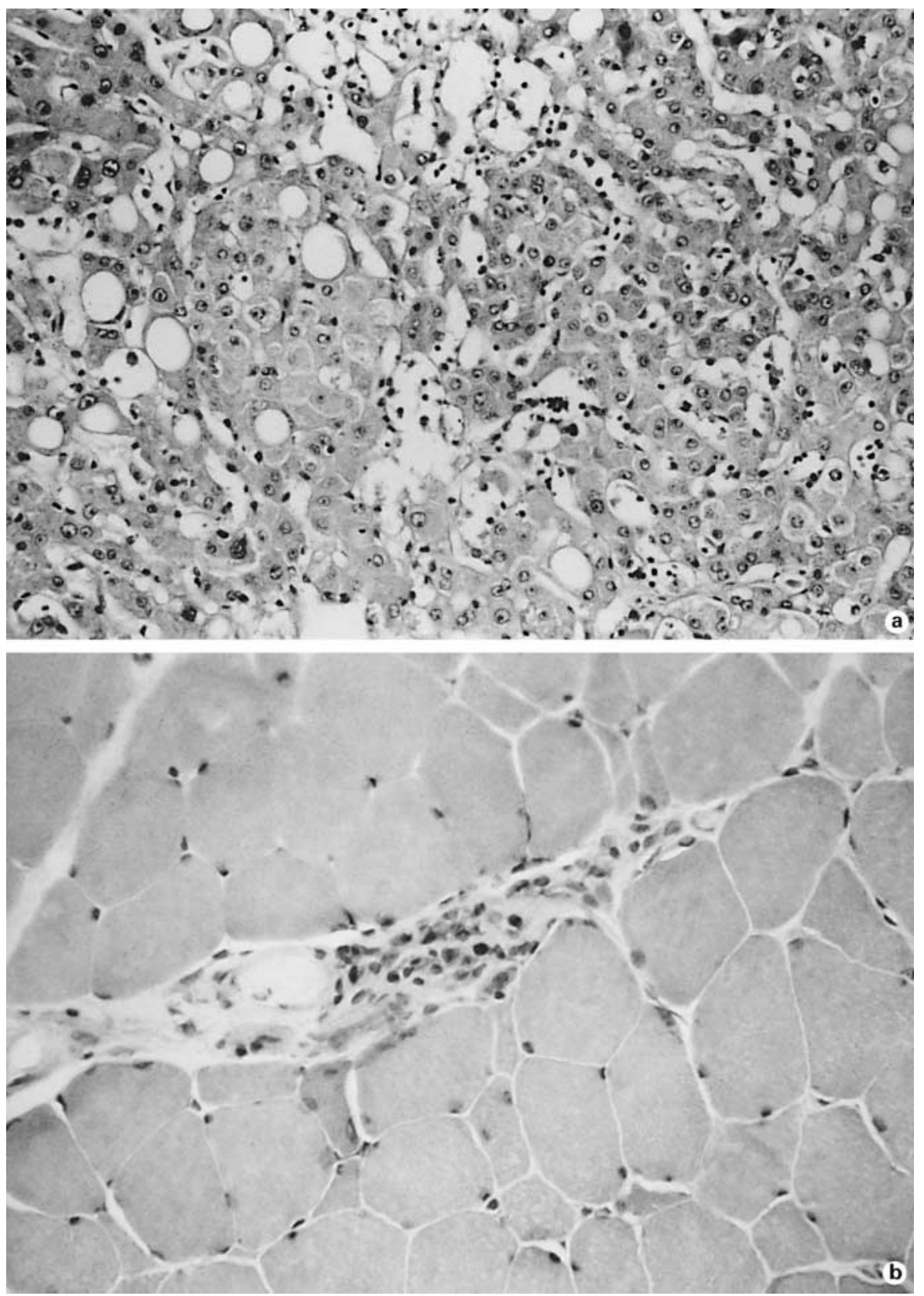

postmenopausal osteoporosis for which she had received hormone replacement therapy for 2 years. She had no history of prior liver disease.

On physical examination, she had well-healed mastectomy scars bilaterally and a limited range of motion in her shoulders. Laboratory studies revealed normal complete blood count, fasting blood sugar, kidney function tests and electrolytes, but elevated transaminases and lactate dehydrogenase (LDH; table 1). Enzyme elevations were evident prior to breast cancer surgery; however, $\gamma$-glutamyltransferase was normal and creatine phosphokinase (CPK) was not available.
Anti-HAV IgM, anti-HBc IgM, HbsAg, HBeAg and anti-HCV IgM, as well as hepatitis $\mathrm{C}$ viral RNA, evaluated by polymerase chain reaction amplification, were negative. Abdominal ultrasonography showed diffuse hepatomegaly, and radiography of the chest was normal. Bone scan revealed increased osteoblastic activity of sacroiliac joints bilaterally.

Her hospital course was complicated by progressive worsening of the enzyme levels in the 2nd week of hospitalization (table 1). Liver biopsy showed parenchymal focal necrosis, sinusoidal congestion and sinusoidal inflammation in addition to mild to moderate mac- 
Fig. 2. Extensive steatosis in the liver.

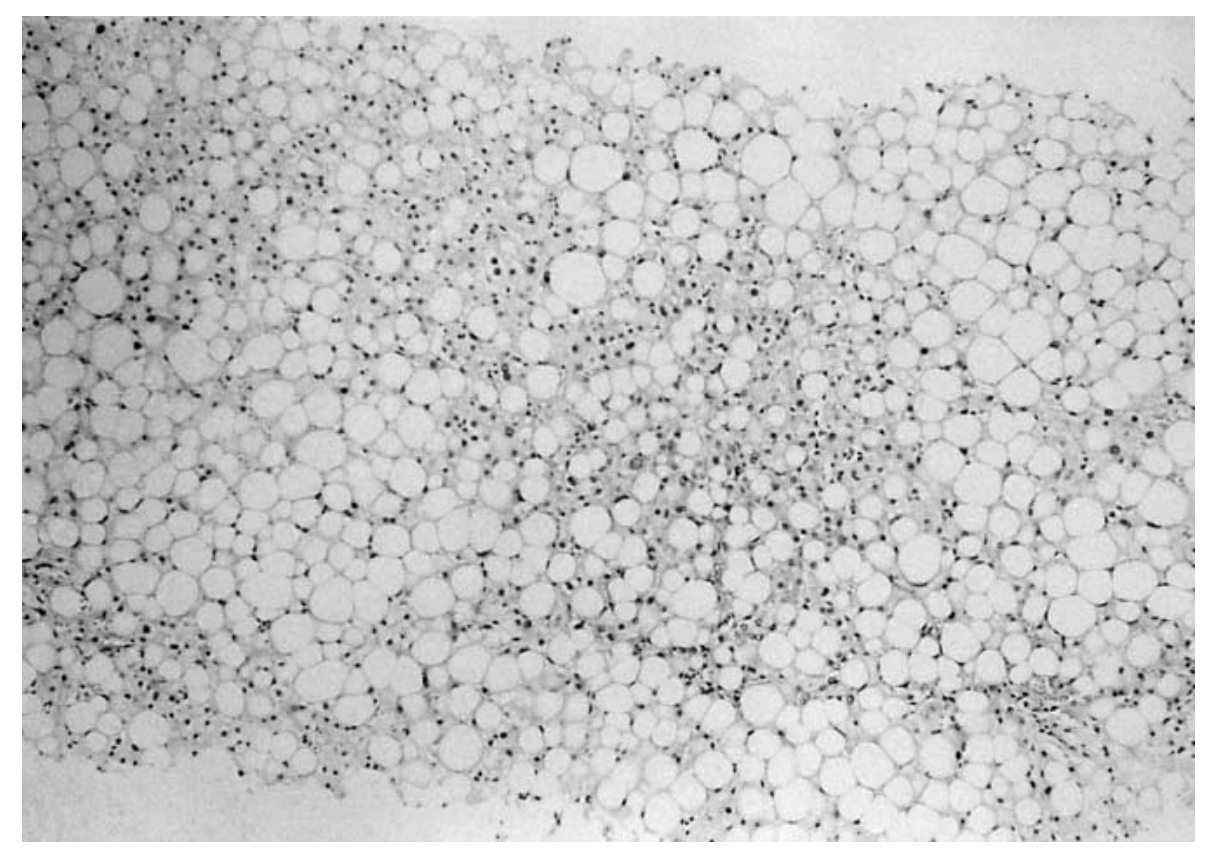

rovesicular steatosis (fig. 1a). Because of mild ductular proliferation with lymphoid infiltration around it, the possibility of a hepatitis C viral infection or autoimmune hepatitis was considered. Antinuclear, liver-kidney microsomal type 1 , smooth muscle and antimitochondrial antibodies were negative. In the $3 \mathrm{rd}$ week of hospitalization, she complained of muscle aches followed by rapidly progressive proximal muscle weakness. Within a few days, she was unable to sit up in bed. CPK was elevated (table 1). Electromyography (EMG) was consistent with myopathy and muscle biopsy revealed myositis characterized by perivascular inflammation, degeneration and regeneration of fibers. There was no perifascicular atrophy (fig. 1b). At this time, there was no evidence of metastasis or accompanying systemic diseases like hyperthyroidism, hypothyroidism, hyperparathyroidism, Cushing's disease, diabetes mellitus or any organ failure. Tumor markers such as CEA and CA 15-3 were within normal limits. She was started on prednisolone $80 \mathrm{mg} /$ day and subsequently treated with 6 courses of cyclophosphamide, methotrexate and 5-fluorouracil (CMF protocol) followed by tamoxifen thereafter.

In the 14th month of follow up, her strength was normal. There was a significant improvement in transaminases and CPK was normal. A repeat liver biopsy showed regression of the necro-inflammatory activity and an increase in steatosis (fig. 2). Steatosis was attributed to glucocorticoid therapy. She developed a compression fracture of the T8 vertebra which was regarded as a complication of glucocorticoid therapy. MRI study did not suggest metastasis. Prednisolone was tapered and discontinued in the 18th month. She remained well for 5 months and then presented with recurrence of proximal muscle weakness associated with elevated enzymes (table 1; 23rd month). Ultrasonography of the abdominal region was normal. She did not respond to high-dose prednisolone therapy and methotrexate was added. In the following month, thoracic X-ray of the patient revealed a newly developed compression fracture of the thoracic spine at T12. Examination of the thoracic spine by MRI revealed tumoral infiltra-
Table 1. Enzyme levels before and after operation

\begin{tabular}{lrrrrrr}
\hline Time & ALT & AST & AP & GGT & LDH & CPK \\
\hline Pre-operative & 229 & 301 & 90 & 50 & 1,044 & N/A \\
Post-operative & & & & & & \\
$\quad$ 2nd week & 258 & 392 & 111 & 107 & 2,013 & N/A \\
1st month & 420 & 775 & 134 & 189 & 3,062 & N/A \\
2nd month & 128 & 268 & 128 & 132 & 1,484 & 3,144 \\
4th month & 37 & 38 & 133 & 52 & 777 & 638 \\
14th month & 31 & 49 & 68 & 78 & 390 & 47 \\
23rd month & 103 & 134 & 65 & 50 & 1,509 & 3,950 \\
27th month & 37 & 177 & 53 & 80 & 1,264 & 391 \\
\hline
\end{tabular}

Elevation of enzymes in the 23rd month was followed by bone metastasis shortly after.

ALT $=$ Alanine transaminase (normal range 0-35 U/1); AST = aspartate transaminase ( $0-35 \mathrm{U} / \mathrm{l}) ; \mathrm{AP}=$ alkaline phosphatase $(75-$ $210 \mathrm{U} / 1) ; \mathrm{GGT}=\gamma$-glutamyltransferase $(5-25 \mathrm{U} / 1) ; \mathrm{LDH}=$ lactate dehydrogenase (100-190 U/1); CPK = creatine phosphokinase $(10$ $70 \mathrm{U} / 1$, for females).

a The levels just prior to start of prednisolone and chemotherapy.

tions on $\mathrm{T} 12$ and $\mathrm{T} 8$ beside the compression fractures. Bone scintigraphy was also consistent with metastasis and showed metastatic activity on $\mathrm{T} 8$ and $\mathrm{T} 12$. At this time, the patient was evaluated by the Departments of Radiation Oncology, Medical Oncology and Neurosurgery. Surgery was not considered and immediate radiotherapy was started. Therefore, biopsy could not been taken. However, after 
Fig. 3. Marked perivesicular collapse in the liver.

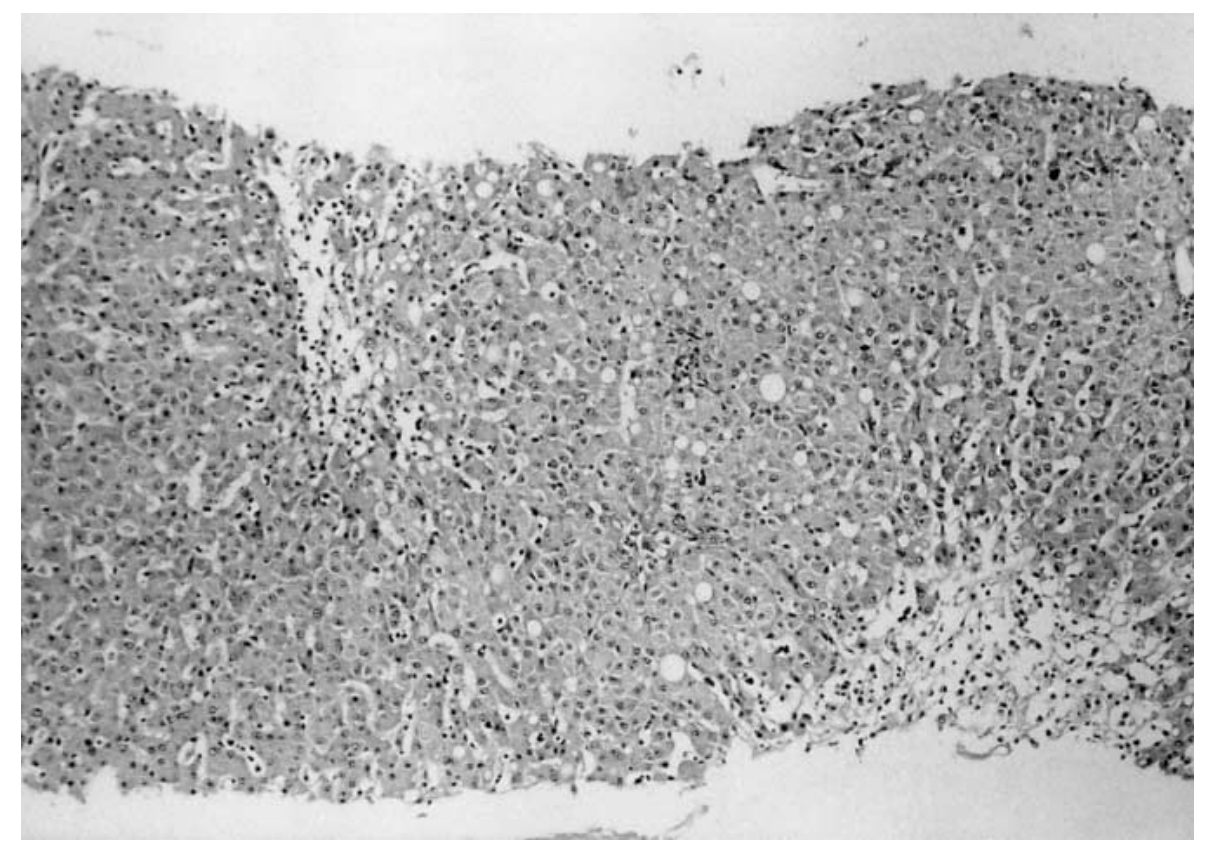

radiotherapy, the patient's symptoms improved. Moreover, her enzymes also improved with radiation and chemotherapy (doxorubucin and cyclophosphamide; table 1; 27th month). However, 10 days after chemotherapy, neutropenic sepsis occurred and the patient died of sudden cardiac arrest. In the postmortem liver biopsy, in addition to mortal tissue changes, there were extramedullary hematopoiesis, mild perivascular congestion and collapse fibrosis probably due to cardiac resuscitation. No other specific or diagnostic features were noted (fig. 3).

\section{Discussion}

Breast cancer associated with both myositis and hepatitis in a patient is presented. The clinical findings, electromyography and muscle biopsy are consistent with myositis. A malignancy may antedate or follow the onset of the myositis by up to 2 years [5]. The clinical course of paraneoplastic myositis has been reported to mirror that of the underlying malignancy [6]. In our case, onset of myositis after removal of the tumor is discordant with the literature; however, the response to adjuvant therapy and steroids suggests a possible relation between the muscle disease and cancer. Beside steroid therapy, adjuvant chemotherapy might have exerted some immunosuppressive effects. Relapse of myositis occurred in the 23rd month, which was followed by bone metastasis shortly after. This also indicates a temporal relationship between the cancer and muscle disease. It can be considered that the etiology of myositis and hepatitis as a paraneoplastic syndrome may be a contradictory suggestion in the absence of a histological confirmation of systemic relapse. At this time, the patient was evaluated by the Departments of Radiation Oncology, Medical Oncology and Neurosurgery. Surgery was not considered and immediate radiotherapy was started. Therefore, biopsy could not been taken. However, after radiotherapy, the patient's symptoms improved. This was a supporting finding for tumor relapse in the patient. Moreover, the findings of the MRI meet all of the metastatic criteria. There were tumoral infiltrations on $\mathrm{T} 12$ and $\mathrm{T} 8$ beside the compression fractures. Bone scintigraphy was also consistent with metastasis and showed metastatic activity on T8 and T12.

It has been suggested that, in patients with malignant diseases, the clinical manifestations of autoimmune rheumatic diseases may either be the result of generation of autoantibodies against various autoantigens including oncoproteins, tumor suppression genes or rheumatic disease associated antigens or they could represent paraneoplastic syndrome or manifest as postchemotherapy rheumatism [7]. In the current case myositis in temporal relation with malignancy following its concurrent remission and relapse suggests a paraneoplastic mechanism.

Hepatitis accompanying polymyositis may raise the question of an association between polymyositis and au- 
toimmune hepatitis as reported before [8]. All viral markers were negative and no causative drug or environmental chemical was evident. Pathological findings of bile duct proliferation associated with lymphoid aggregate formation have been seen in a variety of conditions including viral hepatitis $\mathrm{C}$ disease, primary biliary cirrhosis and autoimmune hepatitis. Since the patient lacked relevant viral serology, the biopsy was interpreted as chronic hepatitis possibly secondary to an autoimmune mechanisms. The presence of accompanying extrahepatic autoimmune syndromes like polymyositis and the response to immunosuppressive treatment are also suggestive and characteristics of autoimmune liver diseases [9].

\section{Conclusion}

Concurrent hepatitis and myositis associated with an underlying malignancy indicate a relationship, possibly via a paraneoplastic mechanism. However, coincidence cannot be excluded. In view of the lack of response to steroid therapy when given alone or with methotrexate added, we can speculate that the autoimmune problem was paraneoplastic in nature and it followed the course of malignancy itself. To the best of our knowledge, hepatitis, which was the leading clinical finding, has not been described as a paraneoplastic syndrome until now and this is the first case of concurrent breast cancer and inflammatory liver and muscle disease reported in the literature.

\section{References}

1 Targoff IN: Polymyositis and dermatomyositis in adults; in Maddison PJ, Isenberg DA, Woo $\mathrm{P}$, Glass DN (eds): Oxford Textbook of Rheumatology, ed 2. Oxford, Oxford University Press, 1998, pp 1249-1287.

2 Zantos D, Zhang Y, Felson D: The overall and temporal association of cancer with polymyositis and dermatomyositis. J Rheumatol 1994; 21:1855-1859.

3 Callen JP: Dermatomyositis and female malignancy. J Surg Oncol 1986;32:121-124.
4 Maoz RC, Langevitz P, Livneh A, Blumstein Z, Sadeh M, Bank I, Gur H, Ehrenfeld M: High incidence of malignancies in patients with dermatomyositis and polymyositis: An 11-year analysis. Semin Arthritis Rheum 1998;27:319324.

5 Tandan R: Dermatomyositis and polymyositis; in Fauci A, Braunwald E, Isselbacher KJ, Wilson JD, Martin JB, Kasoer DL, Hauser SL, Longo DL (eds): Harrison's Principles of Internal Medicine, ed 14. New York, McGraw-Hill, 1998, pp 1896-1901.

6 Verducci MA, Malkasian GD, Friedman SJ, Winkelmann RK: Gynecologic carcinoma associated with dermatomyositis-polymyositis. Obstet Gynecol 1984;64:695-698.
7 Abu-Shakra M, Buskila D, Ehrenfeld M, Con$\operatorname{rad} \mathrm{K}$, Shoenfeld Y: Cancer and autoimmunity: Autoimmune and rheumatic features in patients with malignancies. Ann Rheum Dis 2001;60:433-441.

8 Marie I, Levesque H, Courtois H: Polymyositis, cranial neuropathy, autoimmune hepatitis, and hepatitis C. Ann Rheum Dis 2000;59:839840.

9 Manns MP: Autoimmune hepatitis; in Haubrich WS, Schaffner F, Berk JE (eds): Bochus Gastroenterology, ed 5. Philadelphia, Saunders, 1995, pp 2151-2157. 